\section{Relação Nacional de Medicamentos: uma construção permanente}

The Brazilian National List of Medicines: a work in progress

Lista Nacional de Medicamentos: una

construcción permanente

Rafael Santos Santana 1,2 Izamara Damasceno Catanheide 3

\begin{abstract}
${ }_{1}$ Departamento de Assistência Farmacêutica e Insumos Estratégicos, Ministério da Saúde, Brasília, Brasil.

2 Universidade de Brasília, Brasília, Brasil.

3 Departamento de Gestão e Incorporação de

Tecnologias em Saúde, Ministério da Saúde,

Brasília, Brasil.
\end{abstract}

\section{Correspondência}

\section{R. S. Santana}

Departamento de Assistência Farmacêutica e Insumos Estratégicos, Ministério da Saúde.

Esplanada dos Ministérios, Bloco G, Brasília, DF

70058-900, Brasil.

rafaels.santana1@gmail.com

Sem dúvida, a perspectiva de incorporar a análise de cargas de doenças no processo de elaboração de listas de medicamentos é a grande inovação do artigo Seleção de Medicamentos Essenciais e a Carga de Doença no Brasil 1, a qual entendemos que deveria ser adotada no processo de atualização permanente da Relação Nacional de Medicamentos Essenciais (RENAME). Porém, ao analisar os dados de comparação das diferentes edições da RENAME e o atendimento às cargas de doenças, as autoras interpretam que há um atendimento semelhante ao longo dos anos, no entanto, com a oferta de um número superior de itens na edição de 2012, "sem aparente evidência científica".

Entendemos que os dados apresentados não dão suporte a essa interpretação, pois não houve comparação de dados de evidência entre as diferentes edições da RENAME. Foram apenas analisados o grau de evidência e a força de recomendação de alguns itens da edição de 2012, que ainda assim foram todos considerados "efetivos" ou cuja "evidência favorece a eficácia”. Além disso, também não foi citado qualquer item considerado "não recomendado" pelas análises realizadas 1 .

O texto justifica que o maior número de itens para a mesma carga de doenças "parece ter sido ten- sionada tanto por pressões do crescente mercado de novas tecnologias em saúde como pelas oportunidades políticas de ofertar medicamentos, muitas vezes presentes em ações judiciais" 1 (p. 2351), contudo é necessário ressaltar que a reorientação da atenção à saúde para o atendimento integral do usuário estabelecido pela definição das linhas de cuidado e dos Protocolos Clínicos e Diretrizes Terapêuticas (PCDT) também influenciam a oferta de medicamentos, anteriormente restritos apenas até a "segunda linha de tratamento" 1,2 .

Apesar do texto em vários pontos citar mudanças no processo de atualização da RENAME, em nenhum momento cita que as alterações foram motivadas pela promulgação da Lei no 12.401, de 28 de abril de 2011, que estabelece regras específicas de assistência terapêutica e incorporação tecnológica no SUS ${ }^{3}$. A ausência dessa informação pode induzir o leitor a entender que o processo de seleção de medicamentos em vigor não possui critérios específicos de análise de evidências para a incorporação de novos medicamentos, o que é um erro.

Esse marco legal estabelece como política de Estado para incorporação, alteração e exclusão de medicamentos pelo SUS, a necessidade de realização de processo administrativo, contendo: (1) as evidências científicas sobre a eficácia, a acurácia, a efetividade e a segurança do medicamento; e (2) a avaliação econômica comparativa dos benefícios e dos custos em relação às tecnologias já incorporadas 3 .

Esse processo é realizado pela Comissão $\mathrm{Na}$ cional de Incorporação de Tecnologias no SUS (CONITEC), que reúne em suas análises, especialistas de diversas áreas do cuidado, representantes das diferentes instâncias de gestão do SUS, além de possuir mecanismos de transparência e participação popular por meio de consultas públicas. A instituição de órgãos como esse, dedicado ao trabalho de avaliação de tecnologias em saúde, é uma tendência internacional já bem consolidada em outros países, como Canadá, Reino Unido e Austrália 4,5,6.

As autoras estão corretas ao esclarecer que nessa nova concepção de RENAME houve uma introdução na lista de todos os itens dos componentes de financiamento da Assistência Farmacêutica pactuados anteriormente. E nesse sentido, é possível que haja necessidade de revisão da oferta de alguns medicamentos, conforme pontuado ao longo do texto.

No entanto, é necessário observar que a harmonização das "listas de recomendação" (RENAME anteriores) com as listas de financiamento é o grande avanço trazido pelos novos marcos legais. Essa mudança assegura o acesso dos cidadãos a medicamentos com prévia análise de evidências científicas, e ao mesmo tempo estabelece sua responsabilidade de financiamento por um dos entes da gestão do SUS. Esse último aspecto é inovador e não foi observado na plenitude dos elencos das edições anteriores. Ade- 
mais, a fase atual é marcada pela atualização contínua da lista, num processo transparente de gestão pública, inclusive com a inserção da relação de medicamentos no processo de planejamento e contratualização do SUS 6 .

\section{Colaboradores}

R. S. Santana contribuiu na concepção, revisão e finalização do trabalho. I. D. Catanheide contribuiu na análise, revisão e finalização do trabalho.

1. Figueiredo TA, Schramm JMA, Pepe VLE. Seleção de medicamentos essenciais e carga de doença no Brasil. Cad Saúde Pública 2014; 30:2344-56.

2. Ministério da Saúde. Da excepcionalidade às linhas de cuidado: o Componente Especializado da Assistência Farmacêutica. Brasília: Ministério da Saúde; 2010.

3. Brasil. Lei no 12.401 de 28 de abril de 2011. Altera a Lei no 8.080, de 19 de setembro de 1990, para dispor sobre a assistência terapêutica e a incorporação de tecnologia em saúde no âmbito do Sistema Único de Saúde - SUS. Diário Oficial da União 2011; 29 abr.

4. Banta HD. The development of health technology assessment. Health Policy 2003; 63:121-32.

5. Ministério da Saúde. Avaliação de tecnologias em saúde: ferramentas para a gestão do SUS. Brasília: Ministério da Saúde; 2009.

6. Ministério da Saúde. Balanço Conitec: 2012 2014/Ministério da Saúde, Comissão Nacional de Incorporação de Tecnologias no SUS. Brasília: Ministério da Saúde; 2014.

Recebido em 23/Dez/2014

Aprovado em 14/Jan/2015
As autoras respondem

The authors reply

Las autoras responden

Tatiana Aragão Figueiredo 1

Joyce Mendes de Andrade Schramm 2

Vera Lúcia Edais Pepe 2

${ }_{1}$ Instituto de Tecnologia em Fármacos, Fundação Oswaldo Cruz, Rio de Janeiro, Brasil.

2 Escola Nacional de Saúde Pública Sergio Arouca,

Fundação Oswaldo Cruz, Rio de Janeiro, Brasil.

\author{
Correspondência \\ T. A. Figueiredo \\ Centro Tecnológico de Medicamentos, Instituto de \\ Tecnologia em Fármacos, Fundação Oswaldo Cruz. \\ $A v$. Comandante Guaranys 447, Rio de Janeiro, $R J$ \\ 22775-903, Brasil. \\ tatianafigueiredo@far.fiocruz.br
}

\section{Relação Nacional de Medicamentos: uma (des)construção permanente ou uma confusão crescente?}

As autoras agradecem os comentários ao artigo Seleção de Medicamentos Essenciais e Carga de Doença no Brasil, uma vez que permitem ampliar a discussão em torno da Assistência Farmacêutica vis$\grave{a}$-vis importantes conceitos como essencialidade seleção e incorporação de medicamentos, fundamentais para a construção de um Sistema Único de Saúde (SUS), respeitando seus princípios de equidade, universalidade e integralidade.

O artigo está ancorado, por um lado, no conceito de essencialidade da Organização Mundial da Saúde (OMS) e, por outro, na orientação de que as listas de medicamentos essenciais, nossa brasileira Relação Nacional de Medicamentos Essenciais (RENAME), devem ser construídas baseando-se na seleção de medicamentos respeitando a Carga de Doença do país definida com base em estudos nacionais, e que são a pedra angular, não apenas para a implementação de efetivas Políticas Nacionais de Medicamentos (PNM), mas também para o Uso Racional de Medicamentos.

$\mathrm{O}$ artigo não teve como objetivo analisar o processo de incorporação de novos medicamentos no SUS, que vem sendo realizado pela Comissão Nacional de Incorporação de Tecnologias no SUS (CONITEC), e nem pretendeu questionar a importância de sua existência e de seu trabalho na avaliação de 
tecnologias em saúde. Tampouco pretendeu discutir as mudanças introduzidas na Assistência Farmacêutica pela Lei $n^{\circ} 12.401$, de 28 de abril de 2011, que já foi objeto de comentários anteriormente publicados 1,2 .

$\mathrm{O}$ aumento de 376 medicamentos, selecionados na RENAME 2010, para 810 presentes na RENAME 2012 não se deveu somente à incorporação de novos medicamentos, e sim, à transformação das listas de financiamento da Assistência Farmacêutica em uma lista de medicamentos essenciais. A Carta às Editoras Relação Nacional de Medicamentos: Uma Construção Permanente reconhece que "... As autoras estão corretas ao esclarecer que nessa nova concepção de Rename, houve uma introdução na lista de todos os itens dos componentes de financiamento da Assistência Farmacêutica (...). E nesse sentido, é possível que haja necessidade de revisão da oferta de alguns medicamentos, conforme pontuado ao longo do texto...".

A Carta ilumina algumas "confusões", atualmente existentes, que contribuem para desconstruir o conceito original de essencialidade da OMS: (1) entre a RENAME estabelecida por meio da seleção de medicamentos, considerando as prioridades de saúde da população brasileira e a RENAME resultante da incorporação de medicamentos a serem financiados pelo SUS; e (2) entre lista orientadora da PNM (como recomenda a OMS) e lista orientada pela pactuação de financiamento entre as esferas de governo (fusão dos componentes de financiamento da Assistência Farmacêutica). Essas "confusões" talvez sejam reflexo do que Azeredo 3 (p. 140) identificou sobre aspectos da implementação da nossa PNM, num contexto de reforma gerencialista do setor saúde, em que houve “...o deslocamento do centro do debate da noção de essencialidade, que estruturava o setor (...), para a de incorporação tecnológica que tem recebido maior destaque na estrutura do MS [Ministério da Saúde] nos últimos anos. Outrossim, enquanto a essencialidade era defendida a partir de um horizonte de universalização do acesso, a partir da definição de prioridades, a incorporação tecnológica se erige num discurso de garantidora da integralidade da atenção, $a$ partir do exame das inovações...".

Segundo a OMS ${ }^{4}$ (p. 83), medicamentos essenciais são aqueles “... que satisfazem às necessidades prioritárias no processo do cuidado à saúde de uma população. Eles devem ser selecionados com o objetivo de atender aos problemas de relevância em saúde pública, devendo ser consideradas as evidências de eficácia e segurança, assim como, dados das relações custo-efetividade..." e esse foi o conceito que orientou todas as revisões da RENAME até 2010. Dificilmente uma lista de medicamentos essenciais, utilizando os conceito de essencialidade da OMS, conteria 810 medicamentos, tendo entre eles 5 estatinas (sinvastatina, atorvastatina, fluvastatina, lovastatina e pravastatina), medicamentos da mesma classe terapêutica, mantidos nos elencos das RENAME 2013 e 2014.
No que tange à questão das evidências científicas, o artigo analisou a eficácia, a força de evidência e a força de recomendação para os medicamentos indicados nas principais causas de DALY (disabilityadjusted life years - anos de vida perdidos ajustados por incapacidade), em que havia diferença nos elencos da RENAME 2010 e 2012. A exemplo das estatinas incluídas na RENAME 2012, prevaleceu a inclusão de medicamentos cuja "evidência favorece a eficácia". No mínimo, esses fármacos deveriam ser questionados quanto às evidências científicas que justifiquem sua inclusão em uma lista de medicamentos essenciais. É um bom debate a ser aberto no meio científico.

Bonfim \& Magalhães 2 enumeram as vantagens de uma lista limitada de fármacos de elevada prioridade e apontam seis tipos de divergências existentes entre as RENAME 2010 e 2012. Uma delas é justamente a ampliação dos fármacos de mesma classe terapêutica, sem que haja, na RENAME 2012, informações sobre restrição de uso, favorecendo o uso inadequado destes medicamentos. Outra divergência, para os autores, diz respeito à classificação dos medicamentos na RENAME 2012, cujo critério não é claro, não levando em consideração as necessidades dos prescritores e dispensadores, o que, portanto, não contribui para a "...reorientação da atenção à saúde para o atendimento integral do usuário estabelecido pela definição das linhas de cuidado...", conforme enunciado pela Carta.

Por tudo antes exposto, parece haver, de fato, uma salutar divergência de opiniões uma vez que as autoras não acreditam que a “...harmonização das 'listas de recomendação' (RENAME anteriores) com as listas de financiamento é o grande avanço trazido pelos marcos legais...". Não se discute a importância da existência de ambas, mas sim a substituição de uma pela outra, mantendo-se a mesma denominação para preceitos completamente diferentes. Somos defensoras da RENAME orientadora das PNM e da Assistência Farmacêutica, aderente aos preceitos e conceitos da OMS, respeitando as prioridades de saúde. Não obstante, estudos sobre as listas de medicamentos essenciais, no Brasil, demonstram que, ao transformar a RENAME em lista de financiamento, esta passa a sofrer influências de interesses diferentes daqueles relacionados às necessidades sanitárias, o que pode resultar em sua deturpação 5,6.

Esperamos que este artigo possa contribuir, além da consideração da Carga de Doença na análise e atualização da RENAME, para avivar o importante debate sobre os rumos da Assistência Farmacêutica no Brasil, para que possamos manter os ganhos adquiridos ao longo dos anos, em termos do acesso aos medicamentos, e para que este acesso caminhe na direção do Uso Racional de Medicamentos, beneficiando a saúde da população. 
1. Santos-Pinto CDB, Ventura M, Pepe VLE, Osorio-de-Castro CGS. Novos delineamentos da Assistência Farmacêutica frente à regulamentação da Lei Orgânica da Saúde. Cad Saúde Pública 2013; 29:1056-8.

2. Bonfim JRA, Magalhães PC. Rename 2012: avanço ou retrocesso para a difusão de medicamentos essenciais? Boletim Farmacoterapêutica 2013; (2):9-15.

3. Azeredo TB. Política Nacional de Medicamentos no Brasil: da estrutura normativa à reflexão dos agentes sobre o processo de implementação [Tese de Doutorado]. Rio de Janeiro: Escola Nacional de Saúde Pública Sergio Arouca, Fundação Oswaldo Cruz; 2012.
4. World Health Organization. The selection and use of essential medicines. Report of the WHO Expert Committee (including the 12th Model List of Essential Medicines). http://whqlibdoc.who.int/ trs/WHO_TRS_914_eng.pdf (acessado em 17/ Nov/2012).

5. Pepe VLE, Osorio-de-Castro CGS, Luiza VL. Relação Nacional de Medicamentos Essenciais: um instrumento da Política Nacional de Medicamentos na garantia do acesso. In: Buss PM, Carvalheiro JR, Casas CPR, organizadores. Medicamentos no Brasil: inovação e acesso. v. 1. Rio de Janeiro: Editora Fiocruz; 2008. p. 319-34.

6. Lucchesi G. Dependência e autonomia no setor farmacêutico: um estudo da Ceme [Dissertação de Mestrado]. Rio de Janeiro: Escola Nacional de Saúde Pública, Fundação Oswaldo Cruz; 1991.

Recebido em 06/Fev/2015

Aprovado em 10/Fev/2015 